\title{
Shigellosis with Resultant Septic Shock and Renal Failure
}

\author{
ELBA IRIS MORALEZ, DENENE LOFLAND
}

\begin{abstract}
Septic shock is a rare, potentially lifethreatening complication of bacterial dysentery. The clinical presentation of septic shock includes hypotension, bleeding, hypoxia, acidosis, and jaundice. Historically gram-negative organisms were the most frequent cause of nosocomial bloodstream infections. However isolation of gram-positive organisms has become increasingly frequent with Staphylococcus species accounting for over one half of all nosocomial bloodstream pathogens. Bacterial dysentery is an acute diarrheal illness characterized by abdominal cramping, fever, and the production of mucoid, bloody stools. Laboratory findings include positive stool culture and increased leukocytes in direct fecal exam. Chemistry and hematology values may be abnormal. The disease is usually self-limiting but administration of antibiotics and rehydration therapy may be warranted in severe cases. This case study describes a 53 year old male who presented with diarrhea and diabetic acidosis and subsequently developed respiratory distress and renal failure due to shigellosis. Discussion of disease pathogenesis and treatment are provided.
\end{abstract}

ABBREVIATIONS: $\mathrm{CBC}=$ complete blood count, GI = gastrointestinal, $\mathrm{ER}=$ emergency room, $\mathrm{BUN}=$ blood urea nitrogen, $\mathrm{GFR}=$ glomerular filtration rate, spp. = species, $\mathrm{WBC}=$ white blood cell, LPS = lipopolysaccharide, TLR4 = toll-like receptor 4 , O\&P = ova and parasite, $\mathrm{RBC}=$ red blood cell.

INDEX TERMS: Shigella, septic shock, bacterial dysentery

Clin Lab Sci 2011;24(3):147

Elba Iris Moralez, BS (MT), MLS(ASCP), Trident Hospital System, Charleston, SC
Denene Lofland, PhD, MT(ASCP), Armstrong Atlantic State University, Savannah, GA

Address for Correspondence: Denene Lofland, PhD, MT(ASCP), Armstrong Atlantic State University, Department of Medical Technology, 11935 Abercorn St., Savannah, GA 31419, 912-344-3189, Denene.lofland@ armstrong.edu

\section{Disease Overview}

Shigella is a genus of facultative anaerobic gram-negative bacilli belonging to the bacterial family Enterobacteriaceae. Infection with various species of Shigella may result in a diarrheal illness known as shigellosis or bacterial dysentery.

There are approximately 165 million cases of shigellosis distributed worldwide but most cases occur in developing countries. ${ }^{1}$ The greatest incidence of disease is seen in infants and preschool children. Additional risk factors include poor nutrition and compromised immune status. ${ }^{2}$ Shigellosis is highly infectious, with as few as 10 organisms producing disease in healthy individuals. ${ }^{3}$ It is transmitted through a fecal-oral route or less commonly, through the consumption of contaminated food. It may also be transmitted by flies. Humans and a few primates are the only natural hosts. ${ }^{1}$

Shigellosis, characterized by the production of watery stools, is typically a mild, self-limiting disease that does not require treatment. ${ }^{3,4}$ However, in severe cases, Shigella may invade the intestinal epithelium leading to large scale tissue damage. Severe infections are typically characterized by abdominal cramping, fever, and the production of mucoid, bloody stools. In rare cases or in patients who are immunocompromised, shigellosis may result in severe illness requiring antibiotics and fluid replacement therapy. ${ }^{3}$ 


\section{CLINICAL PRACTICE}

Shigella species (spp.) are isolated from stool culture when the patient is symptomatic. The stool sample is inoculated onto selective agars such as MacConkey and Hektoen Enteric and Selenite Broth. Direct microscopic examination of fecal smears typically demonstrates sheets of white blood cells (WBC). Shigella spp. are rarely isolated from blood culture but when found indicate a particularly invasive and virulent strain. WBC counts may be elevated or decreased but a shift to the left is suggestive of bacillary dysentery. ${ }^{2}$

Encoded within the Shigella genome are several virulence factors which contribute to the pathogenesis of shigellosis, including production of multiple toxins and a type III secretion system. Shigella spp. are capable of producing three toxins: Shigella enterotoxins 1 and 2 and Shiga toxin. The efficacy of the toxins is enhanced through the use of a type III secretion system, which directly injects toxins into the host cell. All three toxins have enterotoxic activity, but Shiga toxin also possesses cytotoxic and neurotoxic activity. Enterotoxic effects are the result of toxins binding to receptors of the small intestine resulting in decreased absorption of electrolytes, glucose and amino acids, while cytotoxic effects result from the inactivation of the 60s ribosomal subunit leading to diminished protein synthesis and ultimately cell death. ${ }^{4}$ The destruction of cells and tissue is a major contributor to the production of watery stools. Neurotoxic effects are often manifested as fever and abdominal cramping.

A rare complication of shigellosis is sepsis, which is defined as a systemic inflammatory response to infection, and is usually attributed to infection of abnormal hosts. ${ }^{5,6}$ Abnormal hosts include those who are immunocompromised, malnourished or suffering from pneumonia, cirrhosis, hepatitis, sickle-cell anemia, or diabetes mellitus. Prior to 1990, gram-negative bacteria were responsible for most cases of sepsis and resulted in significant mortality. The most commonly isolated pathogens were Escherichia coli, Klebsiella and Enterobacter species. ${ }^{7-10}$

Studies conducted during the 1990s found a predominance of gram-positive organism with over onehalf of bloodstream infections due to Staphylococcus species. Gram-negative organisms accounted for approximately one-third of the cases with Eshcerichia coli being isolated most frequently among this group. ${ }^{11,12}$

Today, that trend continues. Studies indicate the incidence of sepsis caused by gram-positive organisms to be as high as $65 \%$. E. coli remains the most commonly isolated gram-negative organism. ${ }^{13-15}$ Table 1 lists the rank order of bloodstream pathogens and their associated mortality. Septicemia due to Shigella is extremely rare $(<1 \%)$ but, when present, the mortality rate is approximately $21 \% .^{11-14,16,17}$

Table 1. Incidence Rate and Mortality of Bloodstream Pathogens*

\begin{tabular}{lcc}
\hline Pathogen & BSI (\%) & Mortality (\%) \\
CoNS $\dagger$ & 31.3 & 20.7 \\
Staphylococcus aureus & 20.2 & 25.4 \\
Enterococcus species & 9.4 & 33.9 \\
Candida species & 9.0 & 39.2 \\
Escherichia coli & 5.6 & 22.4 \\
Klebsiella species & 4.8 & 27.6 \\
Pseudomonas aeruginosa & 4.3 & 38.7 \\
Enterobacter species & 3.9 & 26.7 \\
Serratia species & 1.7 & 27.4 \\
Shigella species & $<1$ & 21.0 \\
\hline
\end{tabular}

* modified from reference 14

$\dagger$ coagulase negative staphylococci

The majority of the systemic effects of sepsis are due to the presence of bacteria within the bloodstream, or bacteremia. Once in the circulation, components of the bacteria cell walls can lead to the activation of complement through both the alternative and classical pathways. ${ }^{18}$ Gram-negative bacterial cell walls contain an immune activating compound known as lipopolysaccharide (LPS), or endotoxin, which binds to surface receptors, such as toll-like receptor 4 (TLR4), expressed by cells of the innate immune system including neutrophils and macrophages. Engagement of TLR4 and other surface receptors results in the production of inflammatory mediators. The inflammatory mediators, which include reactive oxygen species, cytokines, chemokines, prostaglandins and lipid mediators, result in vasodilation, upregulation of adhesion molecules, mobilization and activation of leukocytes. ${ }^{18}$ 


\section{CLINICAL PRACTICE}

The systemic response to endotoxin and bacterial infection leads to damage of multiple organs as a result of infiltration by activated immune cells, including neutrophils. As part of the innate immune system, neutrophils play an important role in the resolution of bacterial infection. Upon activation, neutrophils produce bactericidal compounds, such as reactive oxygen species and proteolytic enzymes, which can eliminate pathogens intracellularly following phagocytosis, or extracellularly following secretion. ${ }^{5}$ In the absence of stimulatory signals, neutrophils have a short circulatory life-span and rapidly undergo programmed cell death or apoptosis. However, in the presence of stimulation, such as engagement of TLR4 by LPS, anti-apoptotic factors, including nuclear factorkappa B, are produced leading to enhanced survival. The increased presence of activated neutrophils in circulation may result in organ damage and even organ failure. ${ }^{5}$ The kidneys, liver, and lungs are the organs most commonly affected by sepsis. In the kidneys, acute tubular necrosis and ultimately acute renal failure may occur due to extracellular damage by lytic factors secreted by neutrophils. Acute renal failure is characterized by increased BUN and creatinine along with decreased glomerular filtration rate (GFR) and oliguria. In the liver resident macrophages, known as Kuppfer cells, play a major role in filtering pathogens and toxins from circulation. ${ }^{18}$ Activation of Kuppfer cells may lead to symptoms of liver damage often observed during sepsis, including bilirubinemia and elevated liver enzymes. In the lungs, damage to lung cells by activated immune cells usually manifests as respiratory alkalosis, hyperventilation, and failure of respiratory muscles. ${ }^{16}$ Hematologically, neutrophils undergo phenotypic changes following activation. The presence of dohle bodies, toxic granulation, and vacuolization are important indicators of bacterial infection and sepsis. ${ }^{16}$

In addition to activation of immune cells, endotoxin can have a significant impact on homeostasis. In the coagulation cascade, endotoxin can activate factor XII of the intrinsic pathway. Increased levels of endotoxin in circulation may result in thrombosis and consumption of platelets and other important factors such as II, V, and VII. The activation of factor XII may also lead to hypotension as factor XII causes the production of bradykinin which increases vascular permeability and decreases vascular resistance. The consumption of platelets and coagulation factors may result in thrombocytopenia and disseminated intravascular coagulation. Additional symptoms of septic patients, include changes in mental status such as disorientation, lethargy, agitation and in diabetic patients, hyperglycemia. ${ }^{16}$

Shigella is rarely associated with bacteremia and occurs mainly in children. We present a case of shigellosis with septic shock in an adult. The patient's underlying condition (diabetes) most likely contributed to the severity of the infection.

\section{Case Study}

A 53 year old male with a history of type II diabetes, hypertension and hyperlipidemia, visited his primary care physician complaining of watery diarrhea lasting six days that was not controlled with over the counter medication. Prior to the onset of symptoms the patient had attended a barbecue and had consumed a cheeseburger from a road-side establishment.

At the time of this visit the patient exhibited a temperature of $100.1^{\circ} \mathrm{F}$. His blood glucose was greater than $500 \mathrm{mg} / \mathrm{dL}$; serum creatinine was $1.8 \mathrm{mg} / \mathrm{dL}$. Complete blood count (CBC) results were reported as normal. Urinalysis results exhibited greater than 1000 $\mathrm{mg} / \mathrm{dL}$ glucose, trace ketones, $1+$ protein, and trace blood.

The patient's hyperglycemia, proteinuria and slight hematuria along with the elevated serum creatinine indicated that the patient may be developing a chronic kidney disease, such as chronic glomerulonephritis, commonly seen in patients with type II diabetes. In addition, gastrointestinal (GI) illness of unknown origin was suspected. However, most food-borne or viral GI infections do not necessitate treatment with antibiotics. The patient was given anti-nausea medication by his primary care physician and advised to increase fluid intake.

Approximately 3 days following his primary care visit, the patient presented to the emergency room (ER) with 


\section{CLINICAL PRACTICE}

diarrhea and weakness lasting 9 days. The patient exhibited an elevated body temperature of $102^{\circ} \mathrm{F}$.

In order to determine the cause of the GI illness, a stool specimen was collected for routine culture along with culture for Yersinia spp., examination for ova and parasites (O\&P), detection of Clostridium difficile toxin and the determination of the presence of WBCs. The stool was negative for $C$. difficile toxin but positive for the presence of many WBCs. The presence of WBCs in the stool specimen indicated possible bacterial dysentery.

The patient was hyperglycemic with high acetone levels, indicating ketoacidosis which is a rare occurrence with type II diabetes. Additionally the patient was suffering from decreased electrolytes and exhibited signs of impaired kidney function as indicated by elevated blood urea nitrogen (BUN) and creatinine along with decreased estimated GFR. Elevated liver enzymes including aspartate aminotransferase and total alkaline phosphatase in conjunction with bilirubinemia also indicated liver damage as shown in Table 2 . The patient was admitted for monitoring of blood glucose levels and was given intravenous normal saline.

On Day 2 of admission, two sets of blood cultures were drawn for routine culture. The patient was showing improved levels of electrolytes and blood glucose. However, the patient was still suffering from hyponatremia and hyperglycemia. Kidney function continued to be impaired as exhibited by oligura, decreased GFR and increased BUN and creatinine. See Table 2 for details. Fluids were increased to achieve a urine output of $30-60 \mathrm{~mL}$ per hour.

Hematological results were abnormal (Table 3). Red blood cells (RBCs) demonstrated an increased mean cell hemoglobin concentration which is consistent with the presence of spherocytes and polychromasia. Anisocytosis, poikilocytosis and nucleated RBCs were present. The WBC differential was also abnormal. There was an increased number of immature neutrophils including all stages of neutrophil development except myeloblasts. Additionally, neutronphils exhibited toxic granulation, dohle bodies, and vacuolization, all of which indicated the presence of an infection. Large platelets were present, but unable to be enumerated due to clumping. Platelet number was estimated to be normal to slightly decreased.

Blood gas results from the evening of Day 2 demonstrated a decreased blood $\mathrm{pH}$ along with decreased bicarbonate indicating metabolic acidosis. Initially, the acidosis was partially and then fully compensated as the partial pressure of carbon dioxide level decreased.

As the evening progressed, the patient's condition deteriorated. The patient experienced respiratory distress and hypotension. Intubation and respiratory assistance from a ventilator were required. In addition, the patient was put on a vasopressor to increase heart rate and blood pressure as a means of circulatory support. At this time it was believed that the patient was experiencing septic shock, however, the causative agent had yet to be identified.

In the early morning hours of Day 3 of admission, chemistry labs demonstrated continued renal failure along with liver dysfunction (Table 2). Urinalysis results indicated acute renal damage demonstrated by the presence of a large amount of protein, RBCs, and granular and WBC casts as shown in Table 4. The presence of bilirubin and urobilinogen indicated liver damage which was also indicated by increased serum bilirubin and liver enzymes.

Both aerobic and anaerobic bottles of the patient's blood culture were shown to be positive for gramnegative bacteria. The bacteria present in the blood cultures were identified as Shigella spp. The same organism was isolated from the stool specimen collected in the ER. The Department of Health and Environmental Control was notified since shigellosis is a reportable disease. Stool cultures were negative for Yersinia and O\&P.

It was determined that the patient experienced acute liver damage, respiratory distress, and acute renal failure secondary to gram-negative sepsis. The patient was placed on a ventilator and administered both a vasopressor for treatment of septic shock and intravenous ceftriaxone to treat shigellosis. 


\section{CLINICAL PRACTICE}

Table 2 Complete Metabolic Panel

\begin{tabular}{lcccc}
\hline & Patient Day 1 & Patient Day 2 & Patient Day 3 & Reference \\
Sodium & 130 & 130 & 135 & $136-145 \mathrm{mEq} / \mathrm{L}$ \\
Potassium & 3.2 & 4.4 & 4.2 & $3.6-5.1 \mathrm{mEq} / \mathrm{L}$ \\
Chloride & 91 & 104 & 107 & $101-111 \mathrm{mEq} / \mathrm{L}$ \\
Carbon Dioxide & 15 & 16 & 17 & $22-32 \mathrm{mEq} / \mathrm{L}$ \\
Glucose & 488 & 251 & 251 & $70-100 \mathrm{mg} / \mathrm{dL}$ \\
Blood Urea Nitrogen & 54 & 49 & 53 & $6-20 \mathrm{mg} / \mathrm{dL}$ \\
Creatinine & 2.6 & 2.0 & 2.8 & $0.7-1.2 \mathrm{mg} / \mathrm{dL}$ \\
Glomerular Filtration Rate & 28 & 37 & 25 & $>60 \mathrm{~mL} / \mathrm{minute}$ \\
Calcium & 8.6 & 7.4 & 6.9 & $8.9-10.3 \mathrm{mg} / \mathrm{dL}$ \\
Total Bilirubin & 2.5 & $*$ & 1.0 & $<1.0 \mathrm{mg} / \mathrm{dL}$ \\
Total Protein & 6.5 & $*$ & 4.4 & $3.1-8.0 \mathrm{~g} / \mathrm{dL}$ \\
Albumin & 2.4 & $*$ & 203 & $<3 / \mathrm{dL}$ \\
Aspartate Aminotransferase & 29 & $*$ & 36 & $32-101 \mathrm{U} / \mathrm{L}$ \\
Total Alkaline Phosphatase & 38 & $*$ & 91 & $10-63 \mathrm{U} / \mathrm{L}$ \\
Alanine Aminotransferase & 35 & $*$ & & $<$ \\
\hline
\end{tabular}

* Test not ordered or not performed

Table 3. Complete Blood Count

\begin{tabular}{|c|c|c|c|c|}
\hline & Patient Day 2 & Patient Day 3 & Patient Day 4 & Reference \\
\hline White Blood Cells & 3.6 & 6.9 & 5.8 & $3.2-11.0109 / \mathrm{L}$ \\
\hline Red Blood Cells & 5.40 & 4.68 & 3.75 & $4.07-5.5810^{12} / \mathrm{L}$ \\
\hline Hemoglobin & 16.4 & 13.5 & 11.3 & $12.9-16.1 \mathrm{~g} / \mathrm{dL}$ \\
\hline Hematocrit & 46.9 & 39.5 & 31.5 & $38.5-47.8 \%$ \\
\hline Mean Cell Volume & 84.6 & 84.5 & 84.1 & $80.9-97.4 \mathrm{fL}$ \\
\hline Mean Cell Hemoglobin & 29.5 & 29.0 & 30.3 & $27-32.9 \mathrm{pg}$ \\
\hline Mean Cell Hemoglobin Concentration & 34.9 & 34.3 & 36.0 & $32.5-34.8 \mathrm{~g} / \mathrm{dL}$ \\
\hline Red Blood Cell Distribution Width & 14.4 & 15.0 & 14.9 & $10.8-14.6 \%$ \\
\hline Platelets & $*$ & * & * & $135-33710^{9} / \mathrm{L}$ \\
\hline Mean Platelet Volume & * & $*$ & * & 7.0-10 fL \\
\hline Neutrophils & 7 & 29 & 58 & $50-70 \%$ \\
\hline Bands & 54 & 50 & 35 & $0-8 \%$ \\
\hline Lymphocytes & 10 & 9 & 5 & $20-50 \%$ \\
\hline Monocytes & 7 & 0 & 2 & $3-10 \%$ \\
\hline Metamyelocyte & 12 & 9 & 0 & $0.00 \%$ \\
\hline Myelocyte & 8 & 2 & 0 & $0.00 \%$ \\
\hline Promyelocyte & 2 & 1 & 0 & $0.00 \%$ \\
\hline Nucleated Red Blood Cells & 2 & 0 & 2 & $0.00 \%$ \\
\hline Polychromasia & present & $\dagger$ & $\dagger$ & absent \\
\hline Poikilocytosis & present & $\dagger$ & present & absent \\
\hline Anisocytosis & present & $\dagger$ & present & absent \\
\hline Microcytes & present & $\dagger$ & $\dagger$ & absent \\
\hline Spherocytes & present & $\dagger$ & $\dagger$ & absent \\
\hline Mega-thrombocytes & present & present & $\dagger$ & absent \\
\hline Absolute Neutrophils & 3.0 & 6.3 & 5.4 & $2.0-7.010^{9} / \mathrm{L}$ \\
\hline Immature/ Total Ratio & 0.92 & 0.68 & 0.38 & $0-0.16$ \\
\hline Toxic Granulation & $1+$ & $2+$ & $1+$ & absent \\
\hline Dohle Bodies & present & present & present & absent \\
\hline Vacuolated Neutrophils & present & present & present & absent \\
\hline Platelet Estimate & normal & normal & normal & normal \\
\hline
\end{tabular}

* Unable to perform due to platelet clumping

$\dagger$ Not observed, category not present 


\section{CLINICAL PRACTICE}

Table 4. Urinalysis (Day 3)

\begin{tabular}{lll}
\hline & Patient & \multicolumn{1}{c}{ Reference } \\
Color & Dark Brown & Clear to yellow \\
Appearance & Cloudy & Clear \\
Glucose & 70 & Negative mg/dL \\
Bilirubin & $1+$ & Negative \\
Ketones & 5 & Negative $\mathrm{mg} / \mathrm{dL}$ \\
Specific gravity & 1.019 & $1.001-1.030$ \\
Blood & $2+$ & Negative \\
pH & 5.0 & $5.0-9.0$ \\
Protein & 100 & Negative $\mathrm{mg} / \mathrm{dL}$ \\
Urobilinogen & 4.0 & $<2.0 \mathrm{mg} / \mathrm{dL}$ \\
Nitrite & Negative & Negative \\
Leukocyte esterase & Trace & Negative \\
Red Blood Cells & $>100$ & $0-2 / \mathrm{hpf}$ \\
White Blood Cells & $3-5$ & $0-2 / \mathrm{hpf}$ \\
Squamous epithelial cells & $0-2$ & Variable/hpf \\
Bacteria & Few & Rare/hpf \\
Hyaline cast & $6-10$ & $0-2 / \mathrm{hpf}$ \\
Granular cast & $>15$ & $0-2 / \mathrm{lpf}$ \\
White Blood Cell Casts & $0-2$ & None/lpf \\
Mucus & None & Variable \\
Amorphous crystals & Many & Variable/hpf \\
& & \\
\hline
\end{tabular}

Two weeks after admission, the patient was discharged from the hospital to a rehabilitation facility. Rehabilitation was required due to muscle weakness that resulted from the long period of bed rest. An oral two-week course of trimethoprim and sulfamethoxazole was prescribed along with nutritional support as the patient was malnourished and anemic subsequent to long-term diarrheal illness. At the time of discharge, the patient was expected to make a full recovery.

\section{REFERENCES}

1. World Health Organization. Guidelines for the control of shigellosis, including epidemics due to Shigella dysenteriae type 1. Geneva, Switzerland: WHO Press; 2005.

2. Mandell GL, Bennett JE, Dolin R, editors. Principles and Practice of Infectious Diseases. $7^{\text {th }}$ ed. Philadelphia: Churchill Livingstone; 2009.

3. Schroeder GN, Hilbi H. Molecular pathogenesis of Shigella spp.: controlling host cell signaling, invasion, and death by type III secretion. Clin. Microbiol. Rev. 2008;21(1):134-56.

4. Niyogi SK, Shigellosis. J. Microbiol. 2005;43(2):133-43.

5. Brown KA, Brain SD, Pearson JD, Edgeworth JD, Lewis SM, Treacher DF. Neutrophils in development of multiple organ failure in sepsis. Lancet. 2006; 368(9530):157-69.
6. Kligler RM, Hoeprich PD. Shigellemia. West. J. Med. 1984; 141(3):375-8.

7. Mandell G, Bennett J, Dolin R, editors. Principles and Practice of Infectious Diseases. $3^{\text {th }}$ ed. Philadelphia: Churchill Livingstone; 1990.

8. Bone R, Fisher C, Clemmer T, Slotman G, Metz G, Balk R. A controlled clinical trial of high-dose methylprednisolone in the treatment of severe sepsis and septic shock. N Engl J Med 1987;317:653-8.

9. Ispahani P, Pearson N, Greenwood D. An analysis of community and hospital-acquired bacteremia in a large teaching hospital in the United Kingdom. Q J Med 1987;241: 427-40.

10. Calandra T, Glauser M, Schellekens J, Verhoef J, the SwissDutch J5 Immunoglobulin Study Group. Treatment of gramnegative septic shock with human IgG antibody to Eschenchia cold J5: a prospeaive, double-blind, randomized study. J Infect Dis $1988 ; 158: 312-9$.

11. Pfaller M, Jones R, Doern G, Kugler K, The SENTRY Participants Group. Bacterial pathogens isolated from patients with bloodstream Infections: frequencies of occurrence and antimicrobial susceptibility patterns from the SENTRY antimicrobial surveillance program (United States and Canada, 1997). Antimicrob Agents and Chemother. 1998;42(7):176270.

12. Edmond M, Wallace S, McClish D, Pfaller M, Jones R, Wenzel R. Nosocomial Bloodstream Infections in Unites States Hospitals: A three year Analysis. Clin. Infect. Diseases. 1999; 29:239-44.

13. Diekema D, Pfaller M, Jones R. The SENTRY Participants Group. Age-related trends in pathogen frequency and antimicrobial susceptibility of bloodstream isolates in North America SENTRY antimicrobial surveillance program, 19972000. International J Antimicrob Agents. 2002;20:412-8.

14. Wisplinghoff H, Bischoff T, Tallent S, Seifert H, Wenzel R, Edmond M. Nosocomial bloodstream infections in US hospitals: analysis of 24,179 cases from a prospective national surveillance study. Clin Infect Disease. 2004;39:309-17.

15. Pien B, Sundaram P, Raoof N, Cost S, Mirrett S, Woods C, Reller L, Weinstein M. The clinical and prognostic importance of positive blood cultures in adults. Amer J Med. 2010;123(9): 819-28.

16. Bone RC. Gram-negative sepsis: a dilemma of modern medicine. Clin. Microbiol. Rev. 1993;6(1):57-68.

17. Struelens M, Pate D, Kabir I, Salam A, Nath S, Butler T. Shigella Septicemia: Prevalence, presentation, risk factors, and outcome. J Infect Dis. 1985;152(4):784-90.

18. Van Amersfoort ES, Van Berkel TJ, Kuiper J. Receptors, mediators, and mechanisms involved in bacterial sepsis and septic shock. Clin. Microbiol. Rev. 2003;16(3):379-414. 